\title{
Analysis with Geographical Detector on the Influencing Factor of Earthquake Mortality in China
}

\author{
Wen Li Jin Chen Wenkai Chen \\ Lanzhou Institute of Seismology, CEA, Lanzhou 730000, China;
}

\begin{abstract}
This paper collected earthquake cases related data in China mainland from 1993 to 2015, we used three geographical detectors (risk detector, factor detector, and interaction detector) based on spatial variation analysis of some potential factors to assess quantitatively their effects on the mortality. It was found that three factors are responsible for mortality: earthquake intensity, house destruction area, and indoor rate. While not observing the population density and the level of economic development in the area of earthquake. There was interaction between factor, mainly for synergies and nonlinear synergies. The social factors enhanced the contribution of natural factors to the mortality rise, and both social factors and natural factors lead to rising the mortality. The study, despite some limitations, has important implications for both researchers and policy makers.
\end{abstract}

Keywords

Earthquake disaster; Mortality; Geographical Detector; Spatial stratified heterogeneity

\section{基于地理探测器的中国地震灾害人员死 亡率影响因素分析}

\author{
李雯 陈晋 陈文凯 \\ 中国地震局兰州地震研究所, 兰州 730000 , 中国
}

摘要: 本文采用中国大陆 1993-2015 年地震灾害损失评估资料, 基于潜在因素的空间分异性 分析, 采用三个地理检测器 (风险检测器、因子检测器和交互检测器) 对地震人员死亡率影 响因素进行定量分析。研究结果表明: 地震烈度、房屋破坏面积、人员在室率是造成地震人 员死亡的三个主要因素, 同时不能忽略地震发生区域的人口密度和经济发展水平的影响; 因 子之间具有交互作用，主要表现为协同作用和非线性协同作用，社会因子增强了自然因子的 贡献量, 共同导致地震人员死亡率的变化。尽管存在一些研究限制, 但对今后地震灾害减灾 和应急救援有着重要作用。

关键词: 地震灾害; 人员死亡率; 地理探测器; 空间分异性

1. 引言 
发生的地震, 从图 1 中不难发现破坏 性地震频繁且强度大、分布广，同时 也具有明显的地区性和成带性, 在空 间上具有迁移性和重复性的特点; 图 2 中显示 1966 年以来每年 5 级以上地震 频次几乎都在 20 次以上, 活动频度较 高。破坏性地震由于其特有的瞬时突 发性特征, 往往会造成严重的生命损 失。据统计 20 世纪后半叶中国大陆不 同自然灾害中, 死亡人数最高的是地 震, 占 $54 \%$, 气象灾害 $40 \%$, 地质灾 害 4\%, 海洋与林业灾害 $1 \%$, 其他灾 害占 $1 \%{ }^{[1]}$ 。为了进一步做好未来预防 措施实现减灾, 需要研究影响地震灾 害人员死亡率的主要因素。

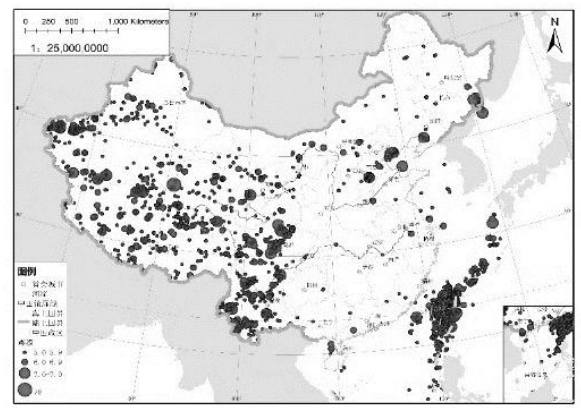

图 11966 年以来中国地震震中分布图 $(M \geq 5)$

Fig 1 China Earthquake epicenter distribution since $1966(\mathrm{M} \geq 5)$

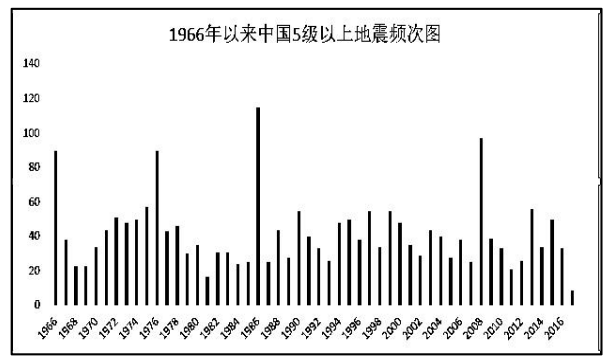

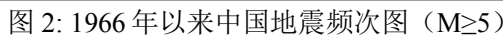

Fig.2 China Earthquake Frequency Chart since 1966 $(\mathrm{M} \geq 5)$

目前地震灾害人员伤亡风险评估 通常使用流行病学方法来衡量风险因 素。例如, 多变量逻辑回归模型用于 分析土耳其地震中的死亡和受伤危险 因素 ${ }^{[2]}$; 基于 AMOS 的地震人员伤亡
影响因素分析, 选择结构方程模型分 析地震人员伤亡影响因素的关系，以 此克服传统分析方法中的局限性 ${ }^{[3]}$; 此 外还有一些研究地震人员伤亡的文献, 但大多都是统计分析的结果, 往往他 们依据的都是震后官方或社会民间的 统计资料，会存在不同程度的误差， 同时造成人员伤亡的因素众多，很有 可能会忽视一些人为或社会因素 ${ }^{[4]}$ 。因 此本文尝试利用地理探测器分析地震 人员死亡率与其影响因子之间的定量 关系, 比较不同影响因子对人员伤亡 率的贡献量大小, 研究影响因子之间 与死亡率的相互关系, 为地震减灾工 作提供科学依据。

\section{2. 研究方法}

地理探测器是探测空间分异性, 揭示其背后驱动力的一组统计学方法 ${ }^{[5]}$, 它既可以探测数值型数据, 也可以探 测定性数据。地理探测器的另一个独 特优势是探测两因子交互作用于因变 量。本文主要采用地理探测器中的风 险探测器、因子探测器和交互作用探 测器对地震灾害人员伤亡的潜在因素 进行空间分异性分析。风险区探测: 通过风险探测器探测统计显著差异的 因子, 确定哪些因子具有风险性, 表 达式如下:

$$
t_{\bar{y}_{h=1}-\bar{y}_{h=2}}=\frac{\bar{Y}_{h=1}-\bar{Y}_{h=2}}{\left[\frac{\operatorname{Var}\left(\bar{Y}_{h=1}\right)}{n_{h=1}}+\frac{\operatorname{Var}\left(\bar{Y}_{h=2}\right)}{n_{h=2}}\right]^{1 / 2}}
$$

式中: $\bar{Y}_{h}$ 表示子区域 $\mathrm{h}$ 内的属性 均值, 如本文中的地震人员死亡率; $n_{h}$ 为子区域 $\mathrm{h}$ 内样本数量, $\operatorname{Var}$ 表示方 差。统计量 $\mathrm{t}$ 近似地服从 Student's $\mathrm{t}$ 分 布, 其中自由度的计算方法为:

$\mathrm{df}$

$$
=\frac{\frac{\operatorname{Var}\left(\bar{Y}_{h=1}\right)}{n_{z=1}}+\frac{\operatorname{Var}\left(\bar{Y}_{h=2}\right)}{n_{h=2}}}{\frac{1}{n_{h=1}-1}\left[\frac{\operatorname{Var}\left(\bar{Y}_{h=1}\right)}{n_{h=1}}\right]^{2}+\frac{1}{n_{h=2}-1}\left[\frac{\operatorname{Var}\left(\bar{Y}_{h=2}\right)}{n_{h=2}}\right]^{2}}
$$


零假设 $H_{0}: \bar{Y}_{h=1}=\bar{Y}_{h=2}$, 如果在置 信水平 $\alpha$ 下拒绝 $H_{0}$, 则认为两个子区域 间的属性值存在着明显的差异。

因子探测器, 探测 $\mathrm{Y}$ 的空间分异 性; 以及探测某因子 $\mathrm{X}$ 多大程度上解 释了属性 $\mathrm{Y}$ 的空间分异。用 $\mathrm{q}$ 值度量 [6]:

$$
\begin{aligned}
& \mathrm{q}=1-\frac{\sum_{h=1}^{L} N_{h} \sigma_{h}^{2}}{N \sigma^{2}}=1-\frac{S S W}{S S T} \\
& \mathrm{SSW}=\frac{\sum_{h=1}^{L} N_{h} \sigma_{h}^{2}}{N \sigma^{2}}, S S T=N \sigma^{2}
\end{aligned}
$$

式中: $h=1, \cdots, L$ 为变量 $Y$ 或因子 $\mathrm{X}$ 的分层（Strata）, 即分类或分区; $N_{h}$ 和 $\mathrm{N}$ 分别为层 $\mathrm{h}$ 和全区的单元数; $\sigma_{h}^{2}$ 和 $\sigma^{2}$ 分别是层 $\mathrm{h}$ 和全区的 $\mathrm{Y}$ 值的方差。

SSW 和 SST分别为层内方差之和和全 区总方差。 $\mathrm{q}$ 的值域为 $[0,1]$ 。交互作 用探测器, 探测风险因子之间是否存 在交互作用:

表 1 两个自变量对因变量交互作用的类型

\begin{tabular}{|c|c|}
\hline 判断 & 交互作用 \\
\hline 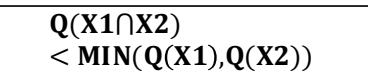 & 非线性减弱 \\
\hline $\begin{array}{l}\operatorname{MIN}(\mathbf{Q}(\mathbf{X 1}), \mathbf{Q}(\mathbf{X 2})) \\
<\mathbf{Q}(\mathbf{X} 1 \cap \mathbf{X} 2) \\
<\operatorname{MAX}(\mathbf{Q}(\mathbf{X 1}), \mathbf{Q}(\mathbf{X 2}))\end{array}$ & $\begin{array}{c}\text { 单因子非线 } \\
\text { 性减弱 }\end{array}$ \\
\hline $\begin{array}{l}\mathbf{Q}(\mathbf{X} 1 \cap X 2) \\
>\operatorname{MAX}(\mathbf{Q}(\mathbf{X} 1), \mathbf{Q}(\mathbf{X} 2))\end{array}$ & 双因子增强 \\
\hline$Q(X 1 \cap X 2)=Q(X 1)+Q(X 2)$ & 独立 \\
\hline $\begin{array}{r}Q(X 1 \cap X 2)>Q(X 1) \\
+Q(X 2)\end{array}$ & 非线性增强 \\
\hline
\end{tabular}
Tab.1 Types of interaction between two covariates

利用地理探测器软件, 以人员死亡率 作为 Grid System 图层, 其他驱动因子 作为地理区域图层, 进行探测分析 ${ }^{[7]}$ 。

\section{3. 数据来源与处理}

\section{1 地震灾害资料收集}

本文系统收集了中国大陆地震灾 害损失评估汇编 [8][9][10][11][12] 、中国 2010 年人口普查分乡、镇、街道资料 [13]、历年中国分省统计年鉴数据, 考 虑地震灾害情况笁选出 1993 年至 2015
年 5 级以上地震 170 例, 涵盖 16 个省, 109 个县, 所选地震空间分布如图 3 所 示。对选取震例的烈度图进行图像配 准、数字化等工作, 得到实际地震烈 度图（矢量图）。由于不同震级的地 震影响的受灾范围是不同的, 而且受 多种因素影响，一般地震 7 度区及以 上烈度区域才会出现人员死亡, 为了 便于统计分析，本文中采用烈度 7 度 区及以上面积为受灾面积, 受灾人口 为 7 度区及以上区域的乡镇人口总数。 基于 GIS 软件平台空间分析，获取受 灾面积及该区域内受灾人口数，从而 求得每次地震的人员死亡率，作为地 理探测器的 Y 因变量。

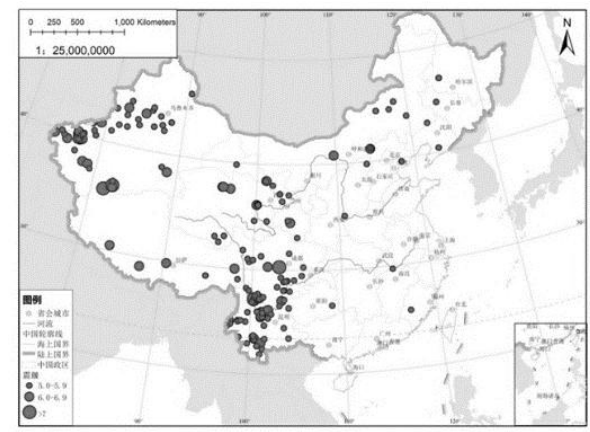

图 3 研究区 1933-2015 年地震震中分布图 $(M \geq 5)$ Fig 3 Earthquake epicenter distribution of the study area from 1933 to $2015(\mathrm{M} \geq 5)$

\section{2 指标选取及其数据来源}

影响地震人员伤亡的关键因素有 地震本身的震级大小、受灾体的天然 环境和社会环境等, 但很难直接确定 这些因素为影响人员伤亡因素 ${ }^{[14]}$ 。实 际上, 确定这些复杂因素通过至少两 个地理层进行操作, 这些地理层通过 GIS 环境来实现。这些层可以分组如下: (1) 自然因素: 地震发生的能量的大 小以及地震动的强度决定了对建筑物 结构的损害 ${ }^{[15]}$; 灾区的地貌类型也可 以影响灾后次生灾害的危险性, 可能 会造成更多的人员死亡。（2）社会因 素: 经济状况影响房屋建造时采用的 
结构特点和建筑材料; 人口聚集程度、 地震发生时人员在室率等因素。这两 类因素细化到 6 个因子: 地震烈度 $(X 1)$ 、人员在室率 $(X 2)$ 、人口密 度（X3）、房屋破坏面积（中等破坏 及以上）（X4）、地貌类型（X5）、 人均 GDP (X6)。

地震烈度根据《中国地震烈度表》 [16]评定， I 度 V 度应以地面上以及 底层房屋中的人的感觉和其他震害现 象为主; $\mathrm{VI}$ 度 $\sim \mathrm{X}$ 度应以房屋震害为 主, XI度和 XII度应综合房屋震害和地 表震害现象。人员在室率的估算采用 肖东升 ${ }^{[17]}$ 基于时空菱形的评估方法。 地貌类型在传统的平原、台地、丘陵 和山地分类的基础上, 按起伏高度对 山地进一步细分, 即划分平原、台地、 丘陵 $(<200 \mathrm{~m})$ 、小起伏山地 $(200 \sim 500 \mathrm{~m})$ 、 中起伏山地 $(500 \sim 1000 \mathrm{~m})$ 、大起伏山地 (1000 2500m) 和极大起伏山地 $(>2500 \mathrm{~m})$ 等 7 个基本地貌 “形态” [18]。房屋破 坏面积数据来自《中国大陆地震灾害 损失评估汇编》（1990 1995）

$$
\text { ( 1996 2000) 、 ( 2001 2005) 、 }
$$$$
\text { （2006 2010）、（2011 2015），选 }
$$
取建筑物中等破坏及以上的面积数据。 乡镇人口及区县人均国民生产总值来 源于中国 2010 年人口普查分乡、镇、 街道资料和 2011 年全国各省统计年鉴

(数据为 2010 年数据)。这些数据包 括因变量 $\mathrm{Y}$ 和自变量数据 $\mathrm{X}$ 。自变量 应为类型量, 如果自变量为数值量, 则需要进行离散化处理。离散可以基 于专家知识, 也可以直接等分或使用 分类算法如 K-means 等 ${ }^{6}$ 。 因变量均已离散化处理。

\section{4. 地震灾害人员死亡率影响因素分析}

\section{1 风险分析}

为了确定各个影响因素对地震人 员死亡的具体影响, 本文从自然因素
和社会因素两个层面开展风险分析。 以不同的地震烈度为例（表 2)，比较 人员死亡率差异性比较。将编号 1,2 , 3 ...对应 I , II , III...上文中已经解释 编号代表类型变量的含义。结果表明 地震烈度按照以上数值进行分级时, 人员死亡率统计显著差异性结果为最 佳, 说明地震烈度对人员死亡具有风 险性。

表 2 不同地震烈度之间人员伤亡率差异性的统计 显著性

Tab.2 Statistical significance of the difference of casualty among different earthquake intensity

\begin{tabular}{ccccc}
\hline $\begin{array}{c}\text { 地震 } \\
\text { 烈度 }\end{array}$ & 6 & 7 & 8 & 9 \\
\hline 6 & & & & \\
\hline 7 & $\mathrm{~N}$ & & & \\
\hline 8 & $\mathrm{Y}$ & $\mathrm{Y}$ & & \\
\hline 9 & $\mathrm{Y}$ & $\mathrm{Y}$ & $\mathrm{N}$ \\
\hline
\end{tabular}

如同地震烈度的风险分析, 对其 他因子作类似分析, 通过风险探测器 反复探测统计显著差异性情况, 根据 探测结果进行最佳分级。探测结果表 明地震烈度因子、建筑物破坏面积因 子均存在差异性, 且差异性显著, 说 明所研究的影响因子对人员死亡具有 风险性, 其他因子影响较弱。

\section{2 因子分析}

利用因子探测器探测影响因子之 间的相对重要性，即每个因子对人口 死亡率的贡献量, 排列顺序如下: 地 震烈度（0.422）>房屋破坏面积 $(0.239)>$ 人员在室率 $(0.040)>$ 人口 密度 $(0.029)>$ 地貌类型 $(0.014)>$ 人 均 GDP (0.013)。

从贡献量大小排序可以看出地震 烈度和房屋破坏面积影响较大。地震 烈度的定义为地震引起的地面震动及 其影响的强弱程度, 表明区域地震烈 度越高遭受地震的影响就越强烈, 人 员死亡率就越高 ${ }^{[19]}$; 房屋破坏面积在 一定程度上代表房屋损毁程度和范围, 
对人员死亡的数量起决定性的作用。 此外人员在室率对地震人员死亡率也 有一定的影响, 其他因素紧随其后, 这些说明社会因素和自然因素不同程 度影响着地震灾害人员死亡率。

\section{3 交互分析}

在自然环境中，地震人员死亡率 是多种因素共同作用的结果，而在实 际环境中, 也不可能存在单一因素或 者单一性质的因素影响人员死亡率的 变化。利用交互探测器探测驱动因子 之间影响人员死亡率的相互关系，驱 动因子之间主要是协同作用和非线性 协同作用，不存在相互独立起作用的 因子（表 3）。

表 3 人员伤亡率影响因子之间的交互作用

Tab. 3 The interaction between the factors of casualty factors

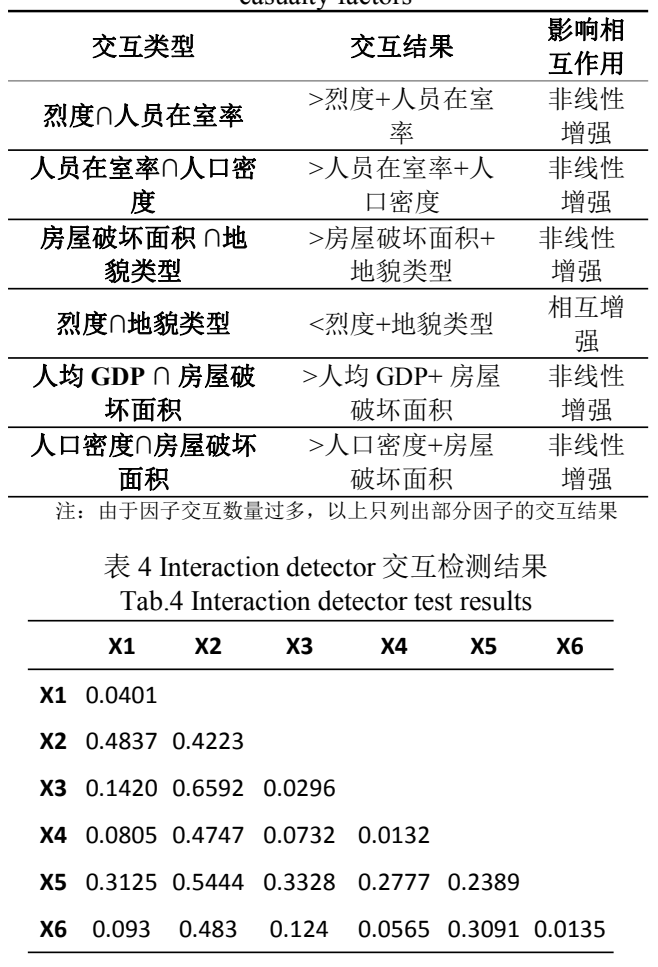

交互检测器可以处理是否两个因 素共同对死亡率的影响比各自独立更 强或更弱。表 4 中人口密度与人均
GDP 交互作用的贡献量与两个因子单 独作用贡献量之和的差值较小, 进一 步说明社会因子之间影响人员伤亡率 的差异性较小。同时社会因子可以增 强自然因子对人员伤亡的贡献量, 即 人类活动对地震人员伤亡的影响越来 越大。这些研究结果表明, 因子之间 的相互作用对地震人员死亡率起重要 作用。

\section{5. 结论}

本文采用地理探测器模型建立地 震人员死亡率 $\mathrm{Y}$ 和风险因子 $\mathrm{X}$ 之间的 关系比经典回归更加精确, 同时探测 两两变量真正的交互作用, 而不限于 计量经济学预先指定的乘性互交, 并 保证了其对自变量共线性免疫。文中 基于潜在因素空间分异性分析, 采用 三个地理检测器评估地震人员伤亡率 的主要影响因素, 研究发现影响地震 人员死亡的因素主要有: 地震烈度、 房屋破坏面积、人员在室率。烈度越 高, 地震的破坏性越大, 导致房屋破 坏等级就越高, 规模就越大, 相应房 屋倒塌率也越高, 倒塌速度加快, 室 内人员存在来不及逃出户外的可能, 因而伤亡越重。其中地震烈度与其他 因素的互动效应比其独立效应更强, 虽然地貌类型、人口密度、人均 GDP 对地震人员死亡率的影响不大, 但与 地震烈度、房屋破坏面积和人员在室 率互相作用对死亡率影响很大, 表明 这三个因素的重要性。同时不能忽略 社会因子人均 GDP 的影响。人均 GDP 水平的高低可以在一定程度上反映该 区域的人均消费水平、教育水平、人 均住房面积等。此外人均纯收入高的 省份, 住房面积较大, 框架结构房屋 比例也相对较大, 总体抗震性能较好, 而在我国西部经济落后地区, 砖混结 构和框架结构房屋比例仍然偏少, 多 以土木、砖木结构为主且抗震性能较 
差。由于部分数据缺失以及数据的不 可用性, 本研究中只对影响地震人员 死亡率的主要风险因子做了定量分析, 量化说明自然因子和社会因子在影响 人员死亡率变化时, 因子之间的相对 重要性以及二者交互作用时影响死亡 率变化的相对重要性, 进一步分析风 险因子之间的相互关系。而对于每个 风险因子具体怎样影响地震人员死亡 率变化, 其作用过程和作用机制有待 进一步研究。

\section{参考文献}

[1] T.L.Holzer, J.C.Savage, 郎从,等. 全球地震死亡人数与人口 $[\mathrm{J}]$. 世界地震译丛, 2013(2):29-45.

[2] Ellidokuz H, Ucku R, Aydin U Y, et al. Risk factors for death and injuries in earthquake: crosssectional study from Afyon, Turkey.[J]. Croatian Medical Journal, 2005, 46(4):613.

[3] 吴将丰, 王海霞. 基于 AMOS 的 地震人员伤亡影响因素分析 [J]. 地震工程与工程振动, 2013, 33(2):221-228.

[4] 赵振东, 郑向远. 地震人员伤亡 研究的回顾与进展 $[J]$. 自然灾 害学报, 2000, 9(1):93-99.

[5] 王劲峰, 徐成东. 地理探测器: 原 理与展望 $[J]$. 地理学报, 2017, 72(1):116-134.

[6] Wang J F, Li X H, Christakos G, et al. Geographical DetectorsBased Health Risk Assessment and its Application in the Neural Tube Defects Study of the Heshun Region, China[J]. International Journal of Geographical Information Science, 2010, 24(1):107-127.

[7] 于佳, 刘吉平. 基于地理探测器 的东北地区气温变化影响因素
定量分析 [J]. 湖北农业科学, 2015, 54(19):4682-4687.

[8] 中国地震局监测预报司. 中国大 陆地震灾害损失评估汇 编:1996-2000[M]. 地震出版社, 2001.

[9] 国家地震局,国家统计局.中国大 陆地震灾害损失评估汇编 (1990-1995 年) [M]. 北京:地震出 版社, 1996.

[10] 楼宝棠. 中国古今地震灾情总汇 [M]. 地震出版社, 1996.

[11] 中国地震局震灾应急救援司. 2006-2010 年中国大陆地震灾害 损失评估汇编 $[M]$. 地震出版社, 2015.

[12] 中国地震局震灾应急救援司. 1966-1989 年中国地震灾害损失 资料汇编 $[M]$. 地震出版社, 2015 .

[13] 中华人民共和国国务院人口普 查办公室. 中国 2010 年人口普 查分乡、镇、街道资料 $[M]$. 中 国统计出版社, 2012.

[14] 张俊玲, 宋莉萍, 钟心, 等. 甘肃 地震造成人员伤亡的要素分析 [J]. 高原地震, 2006, 18(4):62-68.

[15] 田丽莉. 地震灾害人员伤亡影响 因素分析及人员伤亡估算公式 [D]. 首都经济贸易大学, 2012.

[16] 卢寿德. GB/T 17742-2008, 《中 国地震烈度表》, 宣贯教材 $[M]$. 中国标准出版社, 2008.

[17] 肖东升, 常鸣, 苏勇, 等. 基于时 空菱形的人员在室率评估方法 [J]. 世界地震工程, 2016(1):4349.

[18] 李炳元, 潘保田, 韩嘉福. 中国陆 地基本地貌类型及其划分指标 探讨 [J]. 第四纪研究, 2008, 28(4):535-543.

[19] 马玉宏, 谢礼立. 关于地震人员 伤亡因素的探讨 $[J]$. 自然灾害 学报, 2000, 9(3):84-90. 